\title{
Biomimetic nanoparticles enabled by cascade cell membrane coating for direct cross-priming of $\mathrm{T}$ cells
}

\section{Fangjie Chen}

Shanghai Jiao Tong University

\section{Lu Wang}

Shanghai Jiao Tong University

Jinyao Liu ( $\nabla$ jyliu@sjtu.edu.cn )

Shanghai Jiao Tong University https://orcid.org/0000-0002-6044-2033

\section{Article}

Keywords: biomimetic nanoparticles, dendritic cells, T cells

Posted Date: July 15th, 2021

DOI: https://doi.org/10.21203/rs.3.rs-79607/v1

License: (c) (1) This work is licensed under a Creative Commons Attribution 4.0 International License. Read Full License

Version of Record: A version of this preprint was published at Small on November 27th, 2021. See the published version at https://doi.org/10.1002/smll.202104402. 
1 Biomimetic nanoparticles enabled by cascade cell membrane coating

2 for direct cross-priming of $\mathbf{T}$ cells

3

$4 \quad$ Fangjie Chen ${ }^{1}$, Lu Wang $^{1}$, Jinyao Liu ${ }^{1,2 *}$

5

$6 \quad{ }^{1}$ Shanghai Key Laboratory for Nucleic Acid Chemistry and Nanomedicine, Institute of

7 Molecular Medicine, State Key Laboratory of Oncogenes and Related Genes, Shanghai

8 Cancer Institute, Renji Hospital, School of Medicine, Shanghai Jiao Tong University,

9 Shanghai 200127, China.

$10{ }^{2}$ Shanghai Key Laboratory of Gynecologic Oncology, Renji Hospital, School of

11 Medicine, Shanghai Jiao Tong University, Shanghai 200127, China.

12

13 *Corresponding: J.L. (jyliu@ sjtu.edu.cn) 


\section{Abstract}

2 Despite the activation of $\mathrm{T}$ lymphocytes by antigen-presenting cells is responsible for

3 eliciting antigen-specific immune responses, their crosstalking suffers from

4 temporospatial limitations and endogenous influencing factors, which restrict the

5 generation of a strong antitumor immunity. Here, we describe the manipulation of

6 cross-priming of T cells using biomimetic nanoparticles (BNs) enabled by cascade cell

7 membrane coating. BNs are resulted from coating nanoparticulate substrates with cell

8 membranes extracted from dendritic cells (DCs) that are pre-pulsed with cancer cell

9 membrane-coated nanoparticles. With a DC membrane that presents an array of cancer

10 cell membrane antigen epitopes, BNs inherit intrinsic membrane function of DCs.

11 Strikingly, BNs can directly cross-prime T cells and provoke robust yet antigen-specific

12 antitumor responses in multiple mouse models. Combination with clinical anti-

13 programmed death-1 antibodies demonstrates a practical way of BNs to achieve

14 desirable tumor regression and survival rate. This work spotlights the impact of

15 nanoparticles on direct cross-priming of $\mathrm{T}$ cells and supports a unique yet modulate

16 platform for boosting an effective adaptive immunity for immunotherapy. 


\section{Introduction}

2 Increasing evidence has shown that immunotherapy is able to successfully fight various

3 classes of cancer by properly regulating the immune system ${ }^{1,2}$. Training $\mathrm{T}$ lymphocytes

4 with appropriate antigen specificities stands for a central route to promote tumor

5 regression $^{3}$. To elicit an antigen-specific antitumor immunity, the stimulation of

6 cytotoxic T cell populations by professional antigen-presenting cells (APCs) is critical ${ }^{4-}$

$7 \quad$. Delivering antigen and adjuvant into APCs and reinfusing engineered antigen-

8 presenting APCs are two main strategies to stimulate tumor-specific T cell responses ${ }^{7,8}$.

9 However, these approaches inevitably depend on the crosstalking between $\mathrm{T}$ cells and

10 APCs, as cross-priming of T cells relies on surface markers of $\mathrm{APCs}^{9,10}$. Unfortunately,

11 numerous endogenous factors including susceptibility, restricted migration, immune

12 suppression, and metabolic constraints can influence the inherent function of APCs ${ }^{11-}$

$13{ }^{13}$. In addition, the communication between APCs and T cells is often inadequate due

14 to temporal and spatial limitations, resulting in suboptimal antigen presentation and

15 insufficient activation of $\mathrm{T}$ cells ${ }^{14-16}$.

16 To tackle these difficulties, various artificial APCs that mimic the function of

17 endogenous counterparts have been engineered to express proper biological signals,

18 such as major histocompatibility complex (MHC) proteins and costimulatory

19 markers ${ }^{17-19}$. These artificial cells have been successfully exploited for engaging with

20 and activating $\mathrm{T}$ cells ${ }^{20}$. In addition to cell-based artificial APCs, particulate APCs

21 anchoring with requisite ligands including CD28 agonists and epitope-loaded MHC on 
1 their surface have emerged as elegant analogues to facilitate antigen presentation ${ }^{21-24}$.

2 Despite the potential of artificial APCs for adaptive cell-based therapy and the

3 advantage of synthetic particles to penetrate lymph nodes after subcutaneous

4 administration, the construction of artificial systems is complex and time consuming ${ }^{25-}$

$5 \quad 27$. Furthermore, epitope-embedded MHC multimer complexes can be engineered only

6 individual epitope of interest has been identified beforehand ${ }^{28}$. Additionally,

7 introducing certain membrane proteins of APCs can solely confer with partial function

8 of the interaction with T cells, largely limiting their application ${ }^{29}$. Therefore, alternative

9 methods that are facile and versatile yet capable of directly cross-priming $\mathrm{T}$ cells even

10 with an array of unidentified tumor-associated antigen epitopes are highly desirable.

11 Here, we report the use of biomimetic nanoparticles (BNs) enabled by cascade cell

12 membrane coating for directly manipulating cross-priming of $\mathrm{T}$ cells. Cancer cell

13 membranes with an array of membrane antigens are extracted and coated onto a

14 nanoparticulate substrate that can be easily devoured by dendritic cells (DCs) for

15 antigen processing and presentation. DC membranes presenting related antigen

16 epitopes are subsequently acquired and fused onto a nanoparticulate core to form BNs.

17 Exceptionally, BNs enabled by the cascade coating retain the entire membrane

18 composition and intrinsic function of DC membranes. Different from endogenous DCs,

19 direct cross-priming of $\mathrm{T}$ cells by BNs that bypass the need for regular antigen

20 processing and presentation elicits strong antigen-specific $\mathrm{T}$ cell responses. We

21 demonstrate the ability of BNs to boost an extraordinary tumor-specific immunity in 
1 multiple tumor models, including ovalbumin expressing B16 (B16-OVA), HPV E6 and

2 E7-expressing TC-1, and Hepa 1-6 tumor-bearing mice. In combination with a clinical

3 immune checkpoint inhibitor, anti-programmed cell death-1 ( $\alpha$ PD-1) antibodies, BNs

4 evidence a practical path to enhance tumor growth inhibition and improve survival. Our

5 work suggests a simple yet universal platform for the manipulation of the interaction

6 with T cells and supports the potential of engineered materials to directly cross-priming

7 T cells for immunotherapy.

$9 \quad$ Results and discussion

10 Preparation of BNs by cascade cell membrane coating

11 Cell membrane coating, which fuses synthetic cores with natural cell membranes, has

12 emerged as a useful approach to endow coated substrates with foreign functions that

13 are intrinsic unachievable ${ }^{30-32}$. For instance, various nanoparticles coated with

14 erythrocyte membranes exhibit prolonged blood circulation due to the presence of a

15 "don't eat me" signal of CD47-SIRPa on the surface ${ }^{33}$. Furthermore, coated

16 nanoparticles can play as decoys to bind and neutralize toxic molecules since the

17 maintenance of the antigenic characteristics of the source cells, such as macrophage

18 and neutrophils ${ }^{34-37}$. More recently, coating with engineered cancer cell membranes

19 expressing MHC and costimulatory marker CD80 has been explored to mimic DCs for

20 antigen presentation ${ }^{26}$. As primary APCs, DCs execute a vital role for antigen

21 processing and cross-priming of antigen-specific $\mathrm{T}$ cells, which can provoke an 
1 adaptive immunity for cancer treatment ${ }^{38,39}$. However, DC membranes presenting

2 identified antigen epitopes have rarely utilized to coat synthetic cores for stimulating

3 tumor-specific immune responses. To build DC membrane-coated nanoparticles, we

4 developed a technique of cascade cell membrane coating, which could display a set of

5 cancer cell associated peptide epitopes on the surface (Figure 1a). First, cell membranes

6 with multiple antigens were acquired from tumor cells $\mathrm{s}^{40}$ and subsequently fused onto a

7 polymeric nanoparticulate core to form cancer cell membrane-coated nanoparticles

8 (CCNPs). Then, DCs derived from bone marrow (BMDCs) were incubated with

9 CCNPs for antigen processing and presentation. Lastly, membranes displaying a cluster

10 of exclusive tumor-associated antigen epitopes were extracted from CCNPs-pulsed

11 BMDCs and coated onto a polymeric nanoparticulate substrate to generate DC

12 membrane-coated nanoparticles.

13 Transmission electron microscopy (TEM) images showed a clear core-shell

14 structure for CCNPs, in which each poly (lactic-co-glycolic acid) (PLGA)

15 nanoparticulate core was visualized surrounding with a cell membrane coating (Figure

16 1d and Figure S1a). Compared with bare PLGA nanoparticles (Ns), dynamic light

17 scattering (DLS) measurement indicated that the hydrodynamic diameter and surface

18 zeta potential of CCNPs separately increased by $\sim 35 \mathrm{~nm}$ (Figure $1 \mathrm{~b}$ ) and $\sim 6 \mathrm{mV}$ (Figure

19 1c). It was worth noting that the membrane proteins were successfully retained after

20 coating onto PLGA nanoparticles (Figure 1e), which was in good agreement with

21 previous studies ${ }^{1}$. After adding into BMDCs, CCNPs could be internalized rapidly, as 
1 confirmed by confocal laser scanning microscopy (CLSM) images (Figure if and

2 Figure S1b). Fluorescent colocalization analysis indicated that CCNPs were devoured

3 in its entirety by DCs, as verified by a highly overlapping signals from the cores and

4 membrane coatings of the coated nanoparticles. As expected, typical membrane

5 antigens including the overexpressed OVA and main melanoma-associated

6 transmembrane protein glycoprotein 100 (gp100) of B16-OVA cells were processed

7 and presented by BMDCs (Figure $1 \mathrm{~g}-\mathrm{i}$ ). To quantify epitopes presented on the cell

8 membrane, CCNPs with cell membrane proteins (CMP) of 40,60, 80, 120, or $160 \mu \mathrm{g}$

9 were added into $1 \times 10^{6}$ DCs. An amount of $40 \mu \mathrm{g}$ OVA complexed with

10 polyetherimide (PEI-OVA) was cultured as a control ${ }^{41}$. Importantly, OVA epitope

11 presented by CCNPs-matured DCs increased with the quantity of membrane proteins

12 (Figure $1 \mathrm{~h}$ and i). Even pulsed with CCNPs that had a total protein amount of $40 \mu \mathrm{g}$,

13 BMDCs exhibited near 5-times higher OVA epitope level on the surface, in contrast to

14 cells primed with equivalent PEI-complexed OVA. In consideration of the fact that

15 actual quantity of OVA delivered by CCNPs was lower than that of PEI-OVA,

16 enhanced presentation suggested that rather than complexed OVA, antigens embedded

17 in cell membranes could be processed more favorably by DCs. It was noted that

18 incubation with free OVA showed negligible corresponding epitope level on the surface

19 of BMDCs (Figure S1c). These results illustrated that coating nanoparticles with cancer

20 cell membranes represented an efficient way to deliver tumor-associated antigens.

21 Similarly, PLGA nanoparticles coated with DC membranes that were extracted from 
1 B16-OVA cell membrane-coated nanoparticles (termed as BDCNs) appeared a typical

2 core-shell nanostructure (Figure $1 \mathrm{j}$ and Figure S3a). The hydrodynamic diameter and

3 surface zeta potential of BDCNs approximated to those of DC membrane vesicles

4 (Figure 1k and 1). Similar to other cell membrane-coated nanoparticles ${ }^{42}$, the colloidal

5 stabilities of cancer membrane-coated and dendritic cell membrane-coated nanoparticles could maintain up to 14 days in both PBS and pure water (Figure S2).

7 The physicochemical properties of the nanoparticles were summarized in Table S1.

8 Taken together, through translocation of B16-OVA cell membranes onto nanoparticles,

9 a biomimetic structure of cancer cells was occurred for interaction with DCs and 10 subsequent antigen processing and presentation. Take advantage of cascade cell

11 membrane coating, nanoparticles displaying autologous epitopes that were derived

12 from membrane antigens of cancer cells could be accomplished by coating with mature

13 DC membranes.

\section{BNs mediated cross-priming of $T$ cells}

16 We investigated whether BDCNs could execute the function of directly activating T cells in vitro. To study the interaction with T cells, the binding of BNs with the surface

18 receptors of $\mathrm{T}$ cells was analyzed by coculture. $\mathrm{CD}^{+} \mathrm{T}$ cells were isolated from the 19 spleen of C57BL/6 mouse and incubated with BDCNs. To examine the specific 20 adhesion with T cells, both Ns and Ns coated with red blood cell membranes (RBC-Ns) 21 or cell membranes extracted from DCs without pre-pulsing with CCNPs were used as 22 controls. As viewed by confocal imaging, $\mathrm{T}$ cells were surrounded with numerous 23 BDCNs, as shown by fluorescence overlap between the cell membranes and 
1 nanoparticles (Figure 2a and Figure S3b). In comparison with limited non-specific

2 binding of the control nanoparticles, the enhanced fluorescence intensity reflected the

3 specificity of BDCNs to efficiently bind with T cells, which was further supported by

4 flow cytometric analysis (Figure $2 b$ ). The activation of $\mathrm{T}$ cells could be explained by

5 tightly regulated $\mathrm{T}$ cell receptor recognition of antigenic peptides in complex with MHC

6 glycoproteins presented on DC membranes. Then, carboxy fluorescein succinimidyl

7 ester (CFSE) dilution was performed to detect $\mathrm{T}$ cell proliferation. $\mathrm{CD} 8^{+} \mathrm{T}$ cells were

8 incubated individually with Ns, BMDCs and BDCNs. PLGA nanoparticles coated with

9 membranes derived from DCs without pretreatment (DCNs) or with PEI-OVA

10 treatment (ODCNs) were prepared as controls. In striking contrast, BDCNs induced

11 significantly increased proliferation of $\mathrm{CD}^{+} \mathrm{T}$ cells than any other groups. As given in

12 Figure 2c, BDCNs showed $\sim 2-3$ times higher activation of $\mathrm{CD}^{+} \mathrm{T}$ cell proliferation in

13 comparison with those of Ns, BMDCs and DCNs, which confirmed the preferential

14 ability of BDCNs to activate $\mathrm{CD} 8^{+} \mathrm{T}$ cells. To further validate whether BDCNs could

15 initiate activation of antigen-specific T cells, OVA positive $\mathrm{CD} 8^{+} \mathrm{T}$ cells were labelled

16 using OVA (SIINFEKL) tetramer (tet) antibody ${ }^{29}$. Other than nanoparticles coated with

17 membranes that were extracted from DCs without antigen pulsing, BDCNs could

18 directly prime remarkable proliferation of OVA-specific T cells, which was near 10-

19 fold higher than that of DCNs (Figure $2 \mathrm{~d}$ and e). We then employed antigen-specific

20 epitopes based on OVA and gp100 to assess in vivo T cell production after intravenous

21 injection of BDCNs. It was worth mentioning that comparable biodistribution to other

22 commonly used nanoparticles was observed after intravenous injection of BDCNs ${ }^{43}$.

23 As expected, these nanoparticles accumulated in the spleen, a major organ for DCs

24 residence. Moreover, the accumulation of BDCNs in tumor increased with time post-

25 injection extending from 0 to 6 hours, as demonstrated by the increase of fluorescence

26 intensity in the sectioned tumor tissue (Figure S4). Mice were intravenously

27 administered with 3 doses of phosphate buffered saline (PBS), Ns, DCNs, ODCNs, or

28 BDCNs on day 0, 5, and 10, respectively (Figure 2f). Spleen tissues were collected and 
1 homogenized on day 15 to analyze the percentage of antigen-specific T cells by flow

2 cytometry. Expectedly, the induction of OVA (Figure $2 \mathrm{~g}$ and i) and gp100 (Figure $2 \mathrm{~h}$

3 and $\mathrm{j}$ ) positive $\mathrm{T}$ cells by BDCNs was far exceeded all control groups. In addition,

4 significantly elevated levels of Ki67 expression and interferon-gamma (IFN- $\gamma$ )

5 production in $\mathrm{CD}^{+} \mathrm{T}$ cells further confirmed that BDCNs promoted the total

6 proliferation and activation of $\mathrm{T}$ cells (Figure $2 \mathrm{k}-\mathrm{m}$ ). Interestingly, the similar

7 expression of costimulatory markers CD80 and CD86 on DCs indicated that BDCNs

8 had limited influence on DC maturation (Figure S3e and f).

9 The comparison of activation efficacy between DCs pulsed with PEI-OVA and

10 ODCNs was also studied. As shown in Figure S3g and h, mice vaccinated with ODCNs

11 showed significantly elevated levels of $\mathrm{CD} 3^{+} \mathrm{CD} 8^{+}$and OVA-specific $\mathrm{T}$ cells, which 12 separately increased by near 2-times compared with mice administrated with DCs. In 13 order to measure their susceptibility to environmental conditions, BDCNs were 14 incubated with $\mathrm{CD}^{+} \mathrm{T}$ cells in presence of $10 \mathrm{mM}$ lactic acid, a tumor-derived 15 metabolite that can influence dendritic cell activation and antigen expression ${ }^{44}$. 16 Interestingly, BDCNs showed 1.7-fold higher proliferation of $\mathrm{CD}^{+} \mathrm{T}$ cells than that of 17 DCs after incubation for 3 days, demonstrating their less susceptible to the insult of 18 lactic acid (Figure S3c). The insusceptibility of BDCNs was further validated by near 19 1.4-fold higher activation efficacy compared to DCs after 24 hours storage at $4{ }^{\circ} \mathrm{C}$ 20 (Figure S3d). These data implied the superiorities of BNs over living DCs particularly 21 in activation of $\mathrm{T}$ cells, insusceptibility to the tumor microenvironment, and storage 22 condition.

\section{Antigen-specific antitumor responses}

25 We next examined the function of BDCNs in tumor-bearing mice, which were 26 subcutaneously inoculated with B16-OVA in the right hind leg. Mice were daily 27 administered with BDCNs at predetermined time points ${ }^{45-47}$ (Figure 3a). Experimental 
1 endpoint was defined as the tumor size of PBS group exceeded $2000 \mathrm{~mm}^{3}$. Peripheral

2 blood, spleen and tumor were sampled for further analysis. Mice treated with PBS and

3 equivalent nanoparticles of Ns, DCNs, or ODCNs were individually used as controls.

4 Encouragingly, mice administered with BDCNs emerged significantly increased

5 percentages of $\mathrm{CD}^{+}$and $\mathrm{Ki} 67^{+}$in $\mathrm{CD}^{+} \mathrm{T}$ cells in the spleen, which disclosed systemic

6 facilitation of $\mathrm{T}$ cell proliferation (Figure $3 \mathrm{~b}$ and $\mathrm{c}$ ). Moreover, the higher levels of

7 CD80, CD86 and MHC-II in splenic DCs represented a more mature phenotype induced

8 by BDCNs (Figure $3 \mathrm{~d}-\mathrm{f}$ ). On the contrary, decreased $\mathrm{CD} 3^{+} \mathrm{CD} 4^{+} \mathrm{T}$ cells and increased

$9 \mathrm{CD} 8^{+} /$Foxp $^{+} \mathrm{CD}^{2} 5^{+} \mathrm{CD} 4^{+}$ratio in comparison to the control groups of PBS, Ns, DCNs

10 and ODCNs might indicate that immunosuppressive environment was relieved (Figure

$113 \mathrm{~g}$ and S5a). Furthermore, BDCNs could elevate IFN- $\gamma$ production from T cells in the

12 spleen tissue (Figure 3h). Particularly, the increase of OVA tet ${ }^{+} \mathrm{T}$ cells demonstrated

13 that BDCNs elicited immune responses in an antigen-specific manner (Figure 3i).

14 Consistently, appropriate priming of tumor-infiltrating lymphocytes mediated by

15 BDCNs was significantly higher than all the controls (Figure 3j-p). Expectedly,

16 negligible $\mathrm{CD}^{+} \mathrm{CD}^{+} \mathrm{T}$ cells were found in tumor site after vaccination with BDCNs

17 (Figure S5b). The higher concentrations of IFN- $\gamma$ (Figure 3q) and TNF- $\alpha$ (Figure 3r) in

18 serum sampled from BDCNs-treated mice further revealed the elicitation of a robust

19 systemic immune response. Additionally, in contrast to other groups,

20 immunofluorescence staining images depicted more infiltrated $\mathrm{CD}^{+} \mathrm{T}$ cells and

21 upgraded cytokine production (Figure $3 u$ and Figure S5c) in the tumor tissue of 
1 BDCNs-treated mice. Moreover, immunohistochemistry staining images implied

2 improved accumulation of $\mathrm{CD}^{+} \mathrm{T}$ cells and $\mathrm{CD} 8^{+} \mathrm{T}$ cells in tumor site after treatment

3 with BDCNs (Figure S6). The promotion of potent immune responses was further

4 supported by the most efficient inhibition of tumor growth after treatment with BDCNs

5 (Figure 3s, t and Figure S5d). Briefly, BNs could generate a strong antigen-specific

6 antitumor immunity in an aggressive yet OVA-expressing melanoma model.

7 A common tumor model of Hepa 1-6 hepatocellular carcinoma was further

8 developed to verify the generality of BNs for utilization in other cancers without

9 identified antigen epitopes. BNs were prepared by coating PLGA nanoparticles with

10 cell membranes extracted from mature DCs that were pre-pulsed with Hepa 1-6 cell

11 membrane-coated nanoparticles and defined as HDCNs. The tumor-bearing mice were

12 similarly treated, as illustrated in Figure 4a. Consistent with results obtained in B16-

13 OVA tumor model, the percentages of $\mathrm{CD}^{+}$and $\mathrm{Ki}^{+} 7^{+}$in $\mathrm{CD}^{+} \mathrm{T}$ cells (Figure $4 \mathrm{~b}$ and

14 c) and the levels of costimulatory signals CD80, CD86 and MHC-II in DCs were

15 remarkably upgraded in the spleen sampled from mice injected with HDCNs, in

16 comparison with the controls of PBS, Ns, and DCNs (Figure 4d-f). The production of

17 IFN- $\gamma$ from $\mathrm{T}$ cells remained the highest level (Figure $4 \mathrm{~g}$ ), while $\mathrm{CD}^{+} \mathrm{CD}^{+} \mathrm{T}$ cells

18 were downregulated and $\mathrm{CD} 8^{+} / \mathrm{Foxp}^{+} \mathrm{CD} 25^{+} \mathrm{CD} 4^{+}$ratio was upregulated after

19 treatment with HDCNs (Figure 4h and i). Together with the upgraded secretion of TNF-

$20 \alpha$ (Figure $4 \mathrm{j})$ and IFN- $\gamma($ Figure $4 \mathrm{k})$ in the serum, these data claimed that HDCNs could

21 initiate a stronger systemic tumor-specific cytotoxic T lymphocyte (CTL) response in 
1 contrast to the controls. In parallel, the proportions of tumor-infiltrating CTLs (Figure

241 ), $\mathrm{Ki}^{+} 7^{+}$(Figure 4m), DC mature markers (Figure 4n-p), CD8 ${ }^{+} / \mathrm{Foxp}^{+} \mathrm{CD}^{2} 5^{+} \mathrm{CD} 4^{+}$

3 ratio (Figure $4 q$ ) and inflammatory cytokines (Figure $4 \mathrm{t}$ and Figure S7b) were the

4 highest in the HDCNs treatment group, whereas the population of $\mathrm{CD}^{+} \mathrm{CD}^{+} \mathrm{T}$ cells

5 maintained the lowest in all experimental groups (Figure S7a). The tumor growth after

6 treatment with HDCNs retarded notably, with part of the tumors eradicated completely,

7 suggesting the stimulation of a potent antitumor response (Figure $4 \mathrm{r}$ and s). Further

8 supported by haematoxylin eosin (H\&E) staining (Figure S7c), the results found in

9 Hepa 1-6 tumor model reasoned that BNs could be exploited to treat tumors in absence

10 of identified antigens.

12 Cross-validation of BNs-leveraged antitumor immunity

13 We further cross-validated the antitumor immunity in B16-OVA and HPV E6 and E7-

14 expressing TC-1 tumor models to identify the specific and autologous characteristic of

15 BNs. Similarly, BNs were fabricated by fusing PLGA nanoparticles with cell

16 membranes sampled from BMDCs that were pre-treated with TC- 1 cell membrane-

17 coated nanoparticles and abbreviated as TDCNs. Mice inoculated with B16-OVA cells

18 were treated with TDCNs, while TC-1 tumor-bearing mice were inversely injected with

19 BDCNs (Figure 5a). Both PBS and the corresponding BNs were used as controls. In

20 B16-OVA tumor model, the application of TDCNs displayed negligible tumor growth

21 inhibition, which was comparable to that of PBS control (Figure 5b and c). On the 
1 contrary, BDCNs showed notable tumor regression, which resulted in an extension in

2 median survival time by 12 days (Figure 5d). Rather than TDCNs, the distinct potency

3 of BDCNs to inhibit B16-OVA tumor growth explained that the elicitation of an

4 effective tumor-specific immunity depended on the presence of autologous peptide

5 epitopes on the surface. In TC-1 tumor model, BDCNs lost their ability to suppress

6 tumor growth and provided neglectable survival benefit over PBS control (Figure 5e

7 and $\mathrm{f}$ ). As expected, TDCNs slowed the growth of TC-1 tumor and prolonged the

8 survival over the controls of PBS and BDCNs (Figure 5g). Meanwhile, the body weight

9 of the treated mice had no evident fluctuations during the experiment, clarifying the

10 limited side effects of these BNs (Figure S8). Treatment with BDCNs and TDCNs in

11 two cross animal models demonstrated the specificities and personalities of BNs in the

12 initiation of antitumor immune responses.

\section{Combination with clinical $\alpha$ PD-1 antibody}

15 Immune checkpoint blockade by preventing PD-1 on $\mathrm{T}$ cells from binding with 16 programmed death ligand 1 (PD-L1) can activate CTLs to attack cancer cells ${ }^{48-50}$. The 17 use of such inhibitors has elicited durable clinical response and long-term remissions 18 in a fraction of cancer patients ${ }^{51}$. However, a large fraction of patients has failed to 19 respond to these agents, showing a low objective response rate of anti-PD therapy ${ }^{52}$.

20 We speculated that the largely augmented immune responses by direct cross-priming 21 of $\mathrm{T}$ cells could address the problem of anti-PD therapy, particularly in solid tumors. 
1 Therefore, we combined BNs with clinical $\alpha$ PD-1 and evaluated the antitumor

2 responses in a more clinically relevant therapeutic setting. The combined modality was

3 first tested in B16-OVA tumor model. In contrast to $\alpha \mathrm{PD}-1$ alone, indicators of

4 upgraded immune responses associated with $\mathrm{T}$ cells and APCs together with

5 inflammatory cytokine IFN- $\gamma$ both in the spleen (Figure 6a-h) and tumor (Figure 6i-o)

6 warranted extremely positive function of the combination of $\alpha \mathrm{PD}-1$ with BDCNs.

7 Moreover, the splenic and intratumoral $\mathrm{CD}^{+} \mathrm{CD}^{+} \mathrm{T}$ (Figure $6 \mathrm{p}$ and Figure S9a)

8 decreased tremendously, implying the lowered negative effect in immune stimulation.

9 In addition to the priming assay, the strikingly upgraded expressions of IFN- $\gamma$ and TNF-

$10 \alpha$ in the serum demonstrated the robust systemic immune responses induced by the

11 combination treatment (Figure 6q and r). Immunofluorescence images including TNF-

$12 \alpha$ together with IFN- $\gamma$ (Figure 6s) and CD8 (Figure S9b) crowded in the tumor site

13 further validated the promotion of a strong antitumor immunity (Figure S9c). In contrast

14 to the limited beneficial effect from $\alpha \mathrm{PD}-1$ therapy alone, the combination therapy

15 delayed the tumor growth, which accompanied with an extension of median survival

16 period from 25 to 40 days (Figure 6t-v). Hepa 1-6 carcinoma model was further used

17 to evaluate the broad applicability of the combination of BNs with $\alpha$ PD-1 for

18 implementation in other cancers in absence of identified antigen epitopes. As shown in

19 Figure 7, the stimulation of T cells (Figure 7a-d), DC maturation (Figure 7e-g), the

20 secretion of cytokines IFN- $\gamma$ and TNF- $\alpha$ (Figure $7 \mathrm{i}$ and $\mathrm{j}$ ) were greatly improved by the

21 combination of HDCNs and $\alpha \mathrm{PD}-1$, which reversely suppressed the $\mathrm{CD} 3^{+} \mathrm{CD} 4^{+} \mathrm{T}$ cells 
1 (Figure 7h). Different from continuous tumor growth in mice treated with $\alpha \mathrm{PD}-1$, the

2 combination treatment was able to abolish all the tumors (Figure 7k and 1), benefiting

3 from the ability of HDCNs to elicit a powerful antitumor immunity.

4 In summary, we have described the use of BNs enabled by cascade cell membrane

5 coating to manipulate the cross-priming of T cells. BNs have been fabricated through

6 coating PLGA nanoparticles with cell membranes extracted from DCs that have been

7 matured with cancer cell membrane-coated nanoparticles. Due to the maintenance of

8 an intact composition of DC membranes, BNs presenting an array of cancer cell

9 membrane antigen epitopes on the surface can inherit intrinsic membrane function of

10 DCs to cross-prime T cells. Significantly, BNs can directly interact with T cells and

11 have elicited a robust antigen-specific antitumor immunity in multiple mouse models

12 including B16-OVA, HPV E6 and E7-expressing TC-1, and Hepa 1-6 tumors. In

13 combination with clinical $\alpha \mathrm{PD}-1$, BNs have demonstrated a practical way to accomplish

14 desirable tumor regression and survival rate. To this end, treatment efficacy and

15 injection frequency of BNs need to be rationally optimized by using immunoadjuvants

16 or adjusting dosage, particle size, and the loading of antigens for future translation.

17 Beyond the specific use of BNs for cross-priming of $\mathrm{T}$ cells, the direct interaction with

18 other immune cells may inspire new methods that can manipulate immune responses

19 for treating other diseases, such as autoimmune disease, infections and inflammations,

20 studies that are currently ongoing. 


\section{Data availability}

2 The datasets generated and/or analyzed during the study are available from the

3 corresponding authors upon reasonable request.

5 Online Content Methods, along with any additional Extended Data display items and

6 SourceData, are available in the online version of the paper; references unique to these

$7 \quad$ sections appear only in the online paper.

9 References

1. Kroll AV, et al. Nanoparticulate delivery of cancer cell membrane elicits

11 multiantigenic antitumor immunity. Advanced Materials 29, 1018-1026 (2017).

12 2. Zhang Q, et al. Neutrophil membrane-coated nanoparticles inhibit synovial

13 inflammation and alleviate joint damage in inflammatory arthritis. Nature

$14 \quad$ Nanotechnology 13, 1182-1190 (2018).

15 3. Riley RS, June CH, Langer R, Mitchell MJ. Delivery technologies for cancer 16 immunotherapy. Nature Reviews Drug Discovery 18, 175-196 (2019).

17 4. Hu CM, Zhang L, Aryal S, Cheung C, Fang RH, Zhang L. Erythrocyte membrane18 camouflaged polymeric nanoparticles as a biomimetic delivery platform. Proceedings 19 of the National Academy of Sciences of the United States of America 108, 10980-10985 20 (2011). 
1 5. Rao L, et al. Cancer cell membrane-coated upconversion nanoprobes for highly

2 specific tumor imaging. Advanced Materials 28, 3460-3466 (2016).

3 6. Chen Z, et al. Cancer cell membrane-biomimetic nanoparticles for homologous-

4 targeting dual-modal imaging and photothermal therapy. ACS Nano 10, 10049-10057

5 (2016).

6 7. Vijayan V, Uthaman S, Park IK. Cell membrane-camouflaged nanoparticles: a

7 promising biomimetic strategy for cancer theragnostics. Polymers 10, 983 (2018).

8 8. Fang RH, et al. Cancer cell membrane-coated nanoparticles for anticancer

9 vaccination and drug delivery. Nano Lett 14, 2181-2188 (2014).

109 . Schetters STT, et al. Outer membrane vesicles engineered to express membrane-

11 bound antigen program dendritic cells for cross-presentation to CD8 (+) T cells. Acta

12 Biomaterialia 91, 248-257 (2019).

13 10. Balyan R, et al. Correlation of cell-surface CD8 levels with function, phenotype 14 and transcriptome of naive CD8 T cells. Immunology 156, 384-401 (2019).

15 11. Verdijk P, et al. Limited amounts of dendritic cells migrate into the T-cell area of 16 lymph nodes but have high immune activating potential in melanoma patients. Clinical 17 Cancer Research 15, 2531-2540 (2009).

18 12. Chang CC, et al. Defective human leukocyte antigen class I-associated antigen 19 presentation caused by a novel beta2-microglobulin loss-of-function in melanoma cells. 20 Journal of Biological Chemistry 281, 18763-18773 (2006). 
1 13. Cubillos-Ruiz JR, et al. ER stress sensor XBP1 controls anti-tumor immunity by

2 disrupting dendritic cell homeostasis. Cell 161, 1527-1538 (2015).

3 14. Gottfried E, et al. Tumor-derived lactic acid modulates dendritic cell activation and

4 antigen expression. Blood 107, 2013-2021 (2006).

5 15. O'Neill LA, Pearce EJ. Immunometabolism governs dendritic cell and macrophage

6 function. Journal of Experimental Medicine 213, 15-23 (2016).

7 16. Awate S, Babiuk LA, Mutwiri G. Mechanisms of action of adjuvants. Frontiers in $8 \quad$ Immunology 4, 114 (2013).

9 17. Turtle CJ, Riddell SR. Artificial antigen-presenting cells for use in adoptive 10 immunotherapy. Cancer Journal 16, 374-381 (2010).

11 18. Butler MO, Hirano N. Human cell-based artificial antigen-presenting cells for 12 cancer immunotherapy. Immunological Reviews 257, 191-209 (2014).

13 19. Eggermont LJ, Paulis LE, Tel J, Figdor CG. Towards efficient cancer 14 immunotherapy: advances in developing artificial antigen-presenting cells. Trends 15 Biotechnology 32, 456-465 (2014).

16 20. Sunshine JC, Green JJ. Nanoengineering approaches to the design of artificial 17 antigen-presenting cells. Nanomedicine 8, 1173-1189 (2013).

18 21. Rhodes KR, Green JJ. Nanoscale artificial antigen presenting cells for cancer 19 immunotherapy. Molecular Immunology 98, 13-18 (2018).

20 22. Fang RH, Zhang L. Nanoparticle-based modulation of the immune system. Annual 21 Review of Chemical and Biomolecular Engineering 7, 305-326 (2016). 
1 23. Sherman SE, Xiao Q, Percec V. Mimicking complex biological membranes and

2 their programmable glycan ligands with dendrimersomes and glycodendrimersomes.

3 Chemical Reviews 117, 6538-6631 (2017).

4 24. Jiang Y, et al. Engineered cell-membrane-coated nanoparticles directly present

5 tumor antigens to promote anticancer immunity. Advanced Materials, e2001808 (2020).

6 25. Zhang S, et al. Mimicking biological membranes with programmable glycan

7 ligands self-assembled from amphiphilic janus glycodendrimers. Angewandte Chemie

8 International Edition 53, 10899-10903 (2014).

9 26. Liao PC, Boldogh IR, Siegmund SE, Freyberg Z, Pon LA. Isolation of 10 mitochondria from Saccharomyces cerevisiae using magnetic bead affinity purification.

11 PloS One 13, e0196632 (2018).

12 27. Radauer-Preiml I, et al. Nanoparticle-allergen interactions mediate human allergic

13 responses: protein corona characterization and cellular responses. Particle and Fibre

14 Toxicology 13, 3 (2016).

15 28. Yaman S, et al. Melanoma peptide MHC specific TCR expressing T-cell membrane 16 camouflaged PLGA nanoparticles for treatment of melanoma skin cancer. Frontiers in 17 Bioengineering and Biotechnology 8, 943 (2020).

18 29. Luo M, et al. A STING-activating nanovaccine for cancer immunotherapy. Nature

19 Nanotechnology 12, 648-654 (2017).

20 30. Hu CM, et al. Nanoparticle biointerfacing by platelet membrane cloaking. Nature $21 \quad 526,118-121(2015)$. 
1 31. Hu Q, Sun W, Qian C, Wang C, Bomba HN, Gu Z. Anticancer platelet-mimicking

2 nanovehicles. Advanced Materials 27, 7043-7050 (2015).

3 32. Hu CM, Fang RH, Copp J, Luk BT, Zhang L. A biomimetic nanosponge that

4 absorbs pore-forming toxins. Nature Nanotechnology 8, 336-340 (2013).

5 33. Li $\mathrm{H}$, et al. Size dependency of circulation and biodistribution of biomimetic

6 nanoparticles: red blood cell membrane-coated nanoparticles. Cells 8, 881 (2019).

7 34. Ou Z, et al. Macrophage membrane-coated nanoparticles alleviate hepatic

8 ischemia-reperfusion injury caused by orthotopic liver transplantation by neutralizing

9 endotoxin. International Journal of Nanomedicine 15, 4125-4138 (2020).

10 35. Zhang Y, et al. Macrophage-membrane-coated nanoparticles for tumor-targeted

11 chemotherapy. Nano Letters 18, 1908-1915 (2018).

12 36. Meng QF, et al. Macrophage membrane-coated iron oxide nanoparticles for

13 enhanced photothermal tumor therapy. Nanotechnology 29, 134004 (2018).

14 37. Bhattacharyya S, Ghosh SS. Transmembrane TNFalpha-expressed macrophage 15 membrane-coated chitosan nanoparticles as cancer therapeutics. ACS Omega 5, 1572$161580(2020)$.

17 38. Hlavackova E, et al. Dendritic cell-based immunotherapy in advanced sarcoma and 18 neuroblastoma pediatric patients: anti-cancer treatment preceding monocyte harvest 19 impairs the immunostimulatory and antigen-presenting behavior of DCs and 20 manufacturing process outcome. Frontiers in Oncology 9, 1034 (2019). 
1 39. Tan G, Wang Z, Zhang X, Cai Z, Zhang J. Induction of CTLs by DCs pulsed with

2 k-ras mutant peptide on the surface of nanoparticles in the treatment of pancreatic

3 cancer. Oncology Reports 26, 215-221 (2011).

4 40. Ronnie H Fang et al. Cancer cell membrane-coated nanoparticles for anticancer

$5 \quad$ vaccination and drug delivery. Nano Lett 14, 2181-2188 (2014).

6 41. Luo $\mathrm{M}$ et al. A STING-activating nanovaccine for cancer immunotherapy. Nat

$7 \quad$ Nanotechnol 12, 648-654 (2017).

8 42. Qiangzhe Zhang et al. Cellular Nanosponges Inhibit SARS-CoV-2 Infectivity. Nano

$9 \quad$ Lett 20, 5570-5574 (2020).

10 43. Kranz LM et al. Systemic RNA delivery to dendritic cells exploits antiviral defence

11 for cancer immunotherapy. Nature 534, 396-401 (2016).

12 44. Gottfried E et al. Tumor-derived lactic acid modulates dendritic cell activation and

13 antigen expression. Blood 107, 2013-2021 (2006)

14 45. Yunching Chen, Surendar R Bathula, Qi Yang, Leaf Huang. Targeted nanoparticles

15 deliver siRNA to melanoma. J Invest Dermatol. 12, 2790-2798 (2010).

16 46. Diana Anissian et al. Piperine-loaded chitosan-STPP nanoparticles reduce neuronal 17 loss and astrocytes activation in chemical kindling model of epilepsy. Int $\mathbf{J}$ Biol 18 Macromol. 107, 973-983 (2018). 
1 47. Lakeisha A Lewter et al. Slow-sustained delivery of naloxone reduces typical

2 naloxone-induced precipitated opioid withdrawal effects in male morphine-dependent

3 mice. J Neurosci Res. 8 (2020).

4 48. Jiao P, et al. Small molecules as PD-1/PD-L1 pathway modulators for cancer

5 immunotherapy. Current Pharmaceutical Design 24, 4911-4920 (2018).

6 49. Topalian SL, Drake CG, Pardoll DM. Immune checkpoint blockade: a common

7 denominator approach to cancer therapy. Cancer Cell 27, 450-461 (2015).

8 50. $\mathrm{Wu} \mathrm{X}$, et al. Application of PD-1 blockade in cancer immunotherapy.

9 Computational and Structural Biotechnology Journal 17, 661-674 (2019).

10 51. Martin-Romano P, et al. Evidence of pseudoprogression in patients treated with

11 PD1/PDL1 antibodies across tumor types. Cancer Medicine 9, 2643-2652 (2020).

12 52. Cai J, et al. The role of PD-1/PD-L1 axis and macrophage in the progression and 13 treatment of cancer. Journal of Cancer Research and Clinical Oncology 145, 1377$141385(2019)$.

15 53. Qiangzhe Zhang et al. Cellular nanosponges inhibit SARS-CoV-2 infectivity. Nano 16 Lett 20, 5570-5574 (2020).

54. Audrey Arrighi et al. Development of PLGA microparticles with high 18 immunoglobulin G-loaded levels and sustained-release properties obtained by spray19 drying a water-in-oil emulsion. Int J Pharm 566, 291-298 (2019). 
1 This work was financially supported by the National Natural Science Foundation of

2 China (21875135), the Recruitment Program of Global Youth Experts of China

3 (D1410022), the Shanghai Municipal Education Commission-Gaofeng Clinical

4 Medicine Grant Support (20181704, 20191820), and the Innovative research team of

5 high-level local universities in Shanghai (SSMU-ZLCX20180701).

6

$7 \quad$ Author contributions

8 J.L. conceived and designed the experiments. F.C. and L.W. performed all experiments.

9 All authors analyzed and discussed the data. F.C. and J.L. wrote the paper.

10

11 Competing interests

12 The authors declare no competing interests. 
a
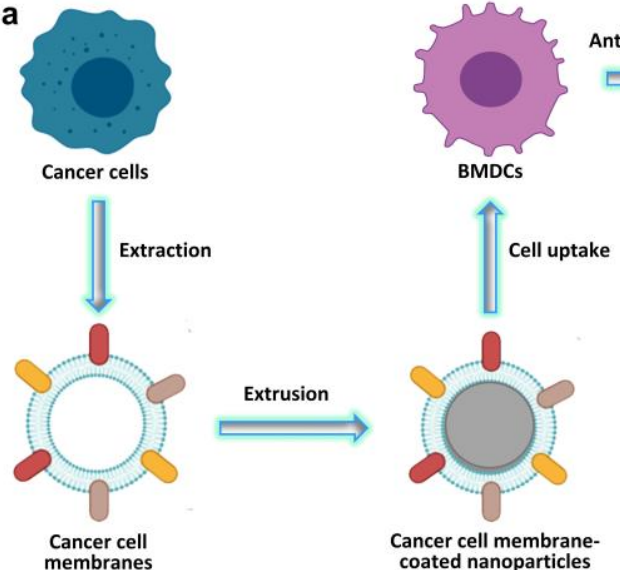
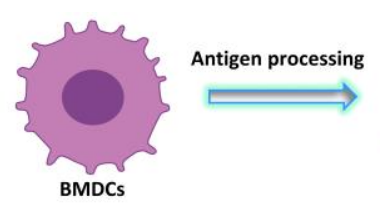

Cell uptake
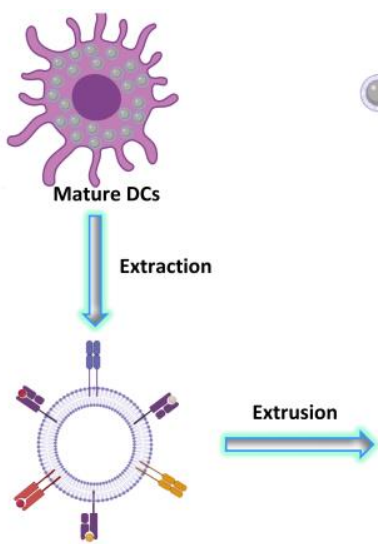

DC membranes

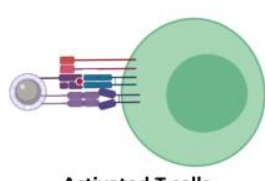

Activated T cells Cross-priming

$\Xi=\mathrm{CD} 80 \quad \Xi-\mathrm{CD} 86$

2- $\mathrm{MHCl}=-\mathrm{MHCl}$

000 Antigens

2 $\mathrm{CD} 28 \equiv \mathrm{TCR} \equiv \mathrm{CD} 8$

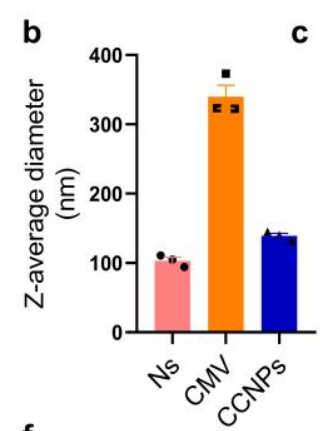

f

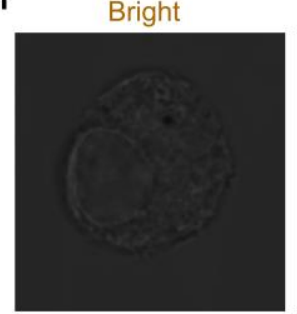

g

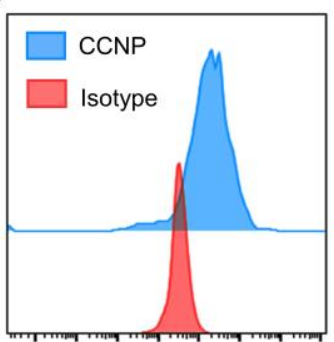

Gp100-FITC

j

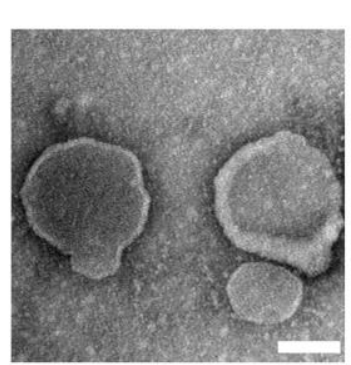

h

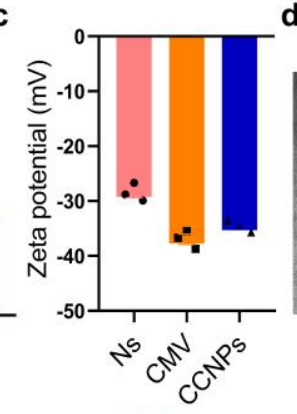

DAPI
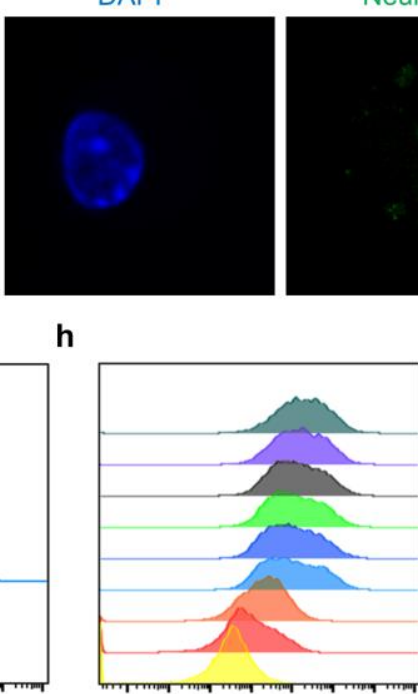

OVAp-H-2kb-APC

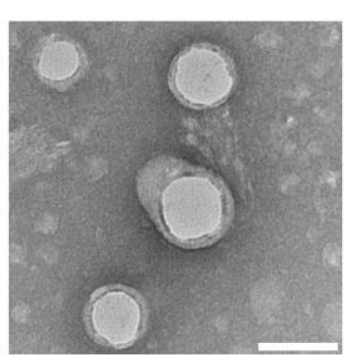

Neuro-DiO
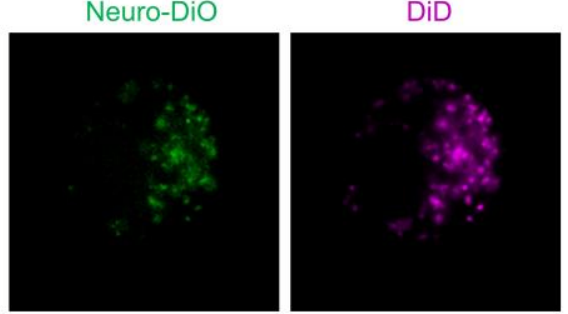

i e

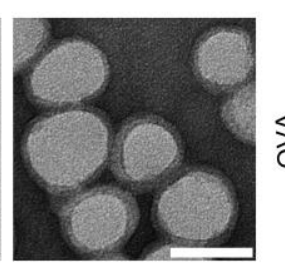

B16-OVA CCNPS lysate

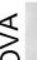

Merge

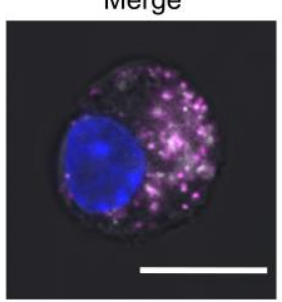

k

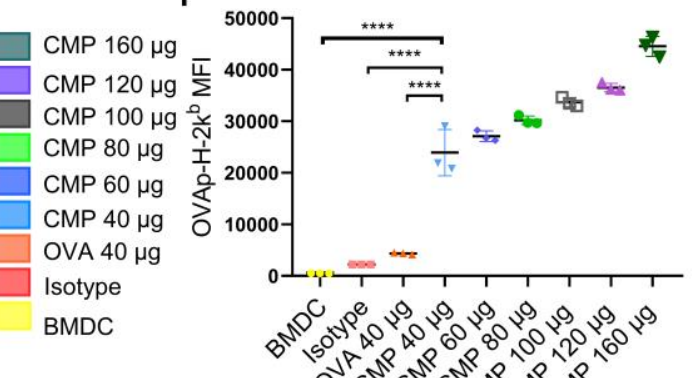

10000
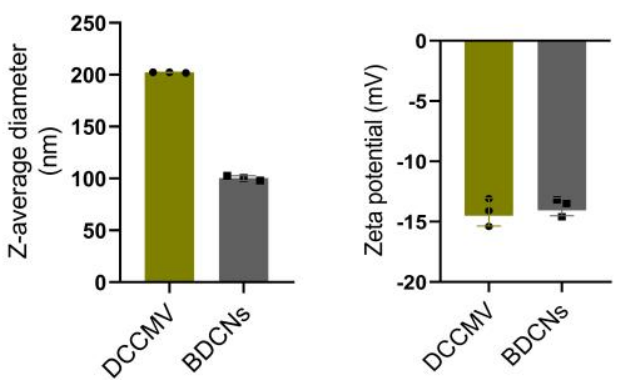
1 Figure 1. Preparation and characterization of BNs. (a) Schematic illustration of BNs enabled by

2 cascade cell membrane coating for direct cross-priming of T cells. (b) Sizes and (c) Zeta potentials 3 of Ns, B16-OVA cell membrane vesicles (CMV), and CCNPs, respectively. CCNPs were prepared 4 by extruding PLGA nanoparticles with cell membranes at a cell membrane protein/nanoparticle weight ratio of 1:1. (d) Typical TEM images of Ns (left) and CCNPs (right) negatively stained with uranyl acetate. Scale bar: $100 \mathrm{~nm}$. (e) Western blot analysis of membrane-specific protein marker OVA on B16-OVA cells and CCNPs, respectively. (f) Colocalization of PLGA cores (DiD, red

8 channel) and cancer cell membranes (Neuro-DiO, green channel) upon BMDC uptake by confocal 9 imaging. Cell nucleus was stained with DAPI (blue channel). Scale bar: $10 \mu \mathrm{m}$. (g) Processing and 10 presentation of antigen gp100 associated with B16-OVA cell membranes by BMDCs. (h) Flow 11 cytometric profiles and (i) mean fluorescence intensities of BMDCs pulsed by CCNPs with different 12 amounts of membrane proteins. BMDCs were stained with OVAp-H-2 $\mathrm{k}^{\mathrm{b}}$-APC. BMDCs, isotype, 13 and PEI-complexed OVA were used as controls. (j) Representative TEM images of BDCNs and DC 14 cell membrane vesicles (DCCMV) negatively stained with uranyl acetate. Scale bar: $100 \mathrm{~nm}$. (k) 15 Sizes and (1) zeta potentials of DC cell membrane vesicles and BDCNs, respectively. BDCNs were 16 prepared by extruding through a polycarbonate membrane with a pore size of $200 \mathrm{~nm}$. Error bars 17 represent the standard deviation $(n=3)$. 

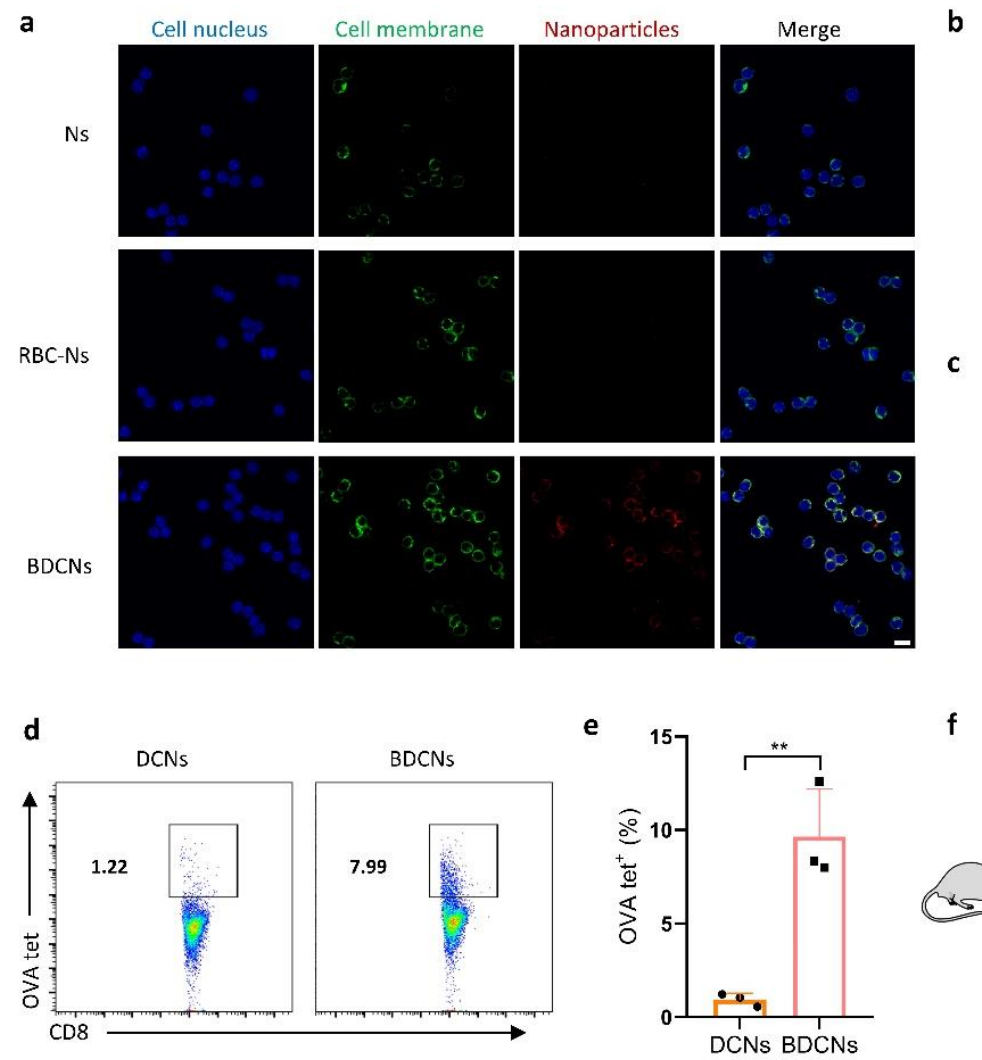

f

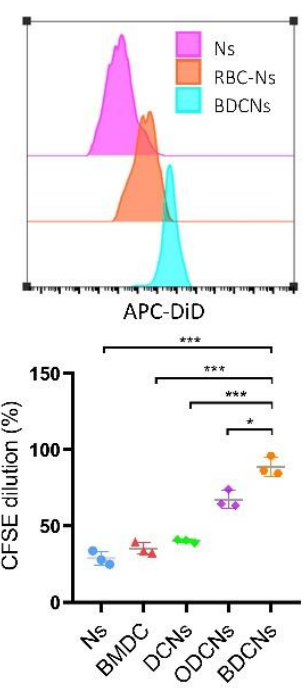

Day 15

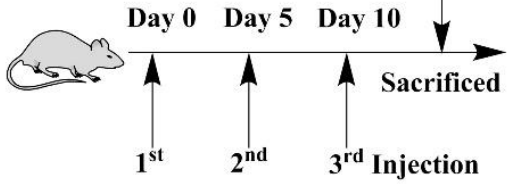

ODCNs

BDCNs
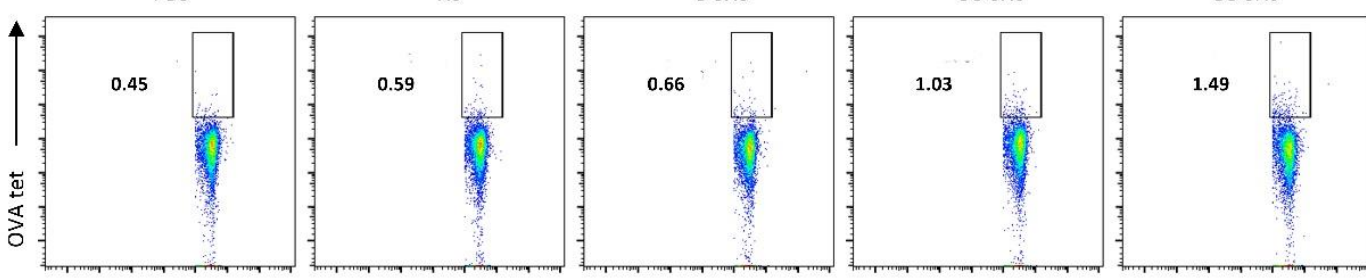

h
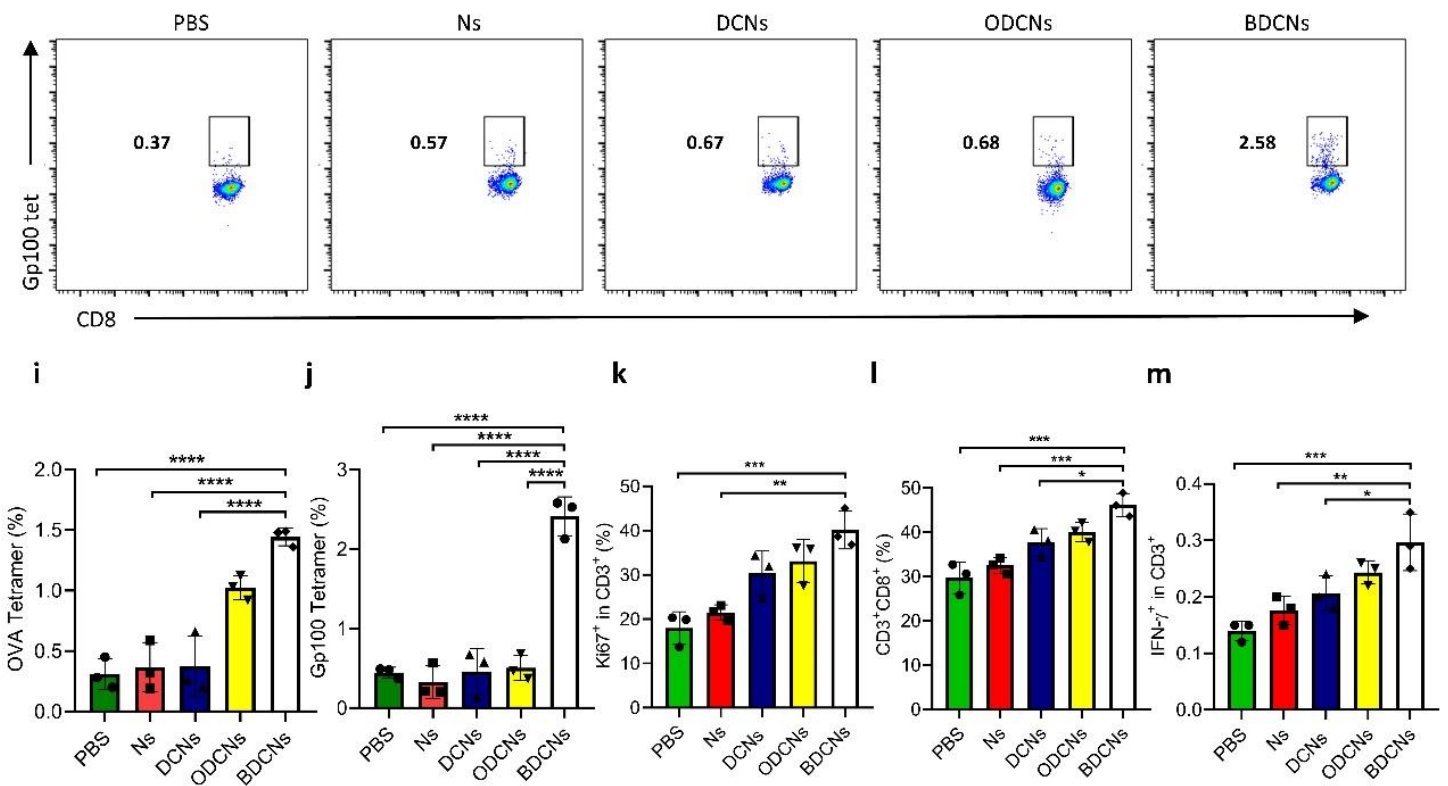

2 Figure 2. Direct cross-priming of T cells by BNs. (a) Representative confocal images and (b) flow

3 cytometric analysis of primary $\mathrm{CD} 8^{+} \mathrm{T}$ lymphocytes after incubation with Ns, RBC-Ns or BDCNs 
1 at $4{ }^{\circ} \mathrm{C}$ for 1-hour. Cell nucleus, cell membrane, and BDCNs were separately stained with Hochest 2 (blue channel), Neuro-DiO (green channel), and DiD (red channel). Scale bar: $10 \mu \mathrm{m}$. (c) Flow 3 cytometric analysis of CFSE-labeled primary $\mathrm{CD}^{+} \mathrm{T}$ cells cultured with Ns, BMDCs, DCNPs, 4 ODCNs or BDCNs. CFSE dilution was used to quantify the proliferation of $\mathrm{CD}^{+} \mathrm{T}$ cells. (d) 5 Representative FACS scatter plots and (e) frequency of SIINFEKL-tetramer CD8 ${ }^{+} \mathrm{T}$ cells in 6 primary splenocytes after incubation with DCNs or BDCNs. (f) Experimental design for in vivo 7 evaluation of $\mathrm{T}$ cell activation by injection with BNs in 6-8 weeks old male C57BL/6 mice. 8 Representative FACS scatter plots and frequency of (g, i) H-2K $\mathrm{K}^{\mathrm{b}}$ OVA Tetramer-SIINFEKL and ( $\mathrm{h}$, 9 j) H-2Db gp100 Tetramer-EGSRNQDWL CD8 ${ }^{+} \mathrm{T}$ cells in splenocytes collected from mice treated 10 with PBS, Ns, DCNs, ODCNs, and BDCNs, respectively. (k) Ki67 $7^{+}$, (l) $\mathrm{CD}^{+}$and (m) $\mathrm{IFN}-\gamma^{+}$in $11 \mathrm{CD}^{+}$of splenocytes from the treated mice. Error bars represent the standard deviation $(n=3)$. 12 Significance was assessed using One-way ANOVA with Dunnett's post-hoc test. $* * * * p<0.0001$, $13 * * * p<0.001, * * p<0.01$, and $* p<0.05$. 


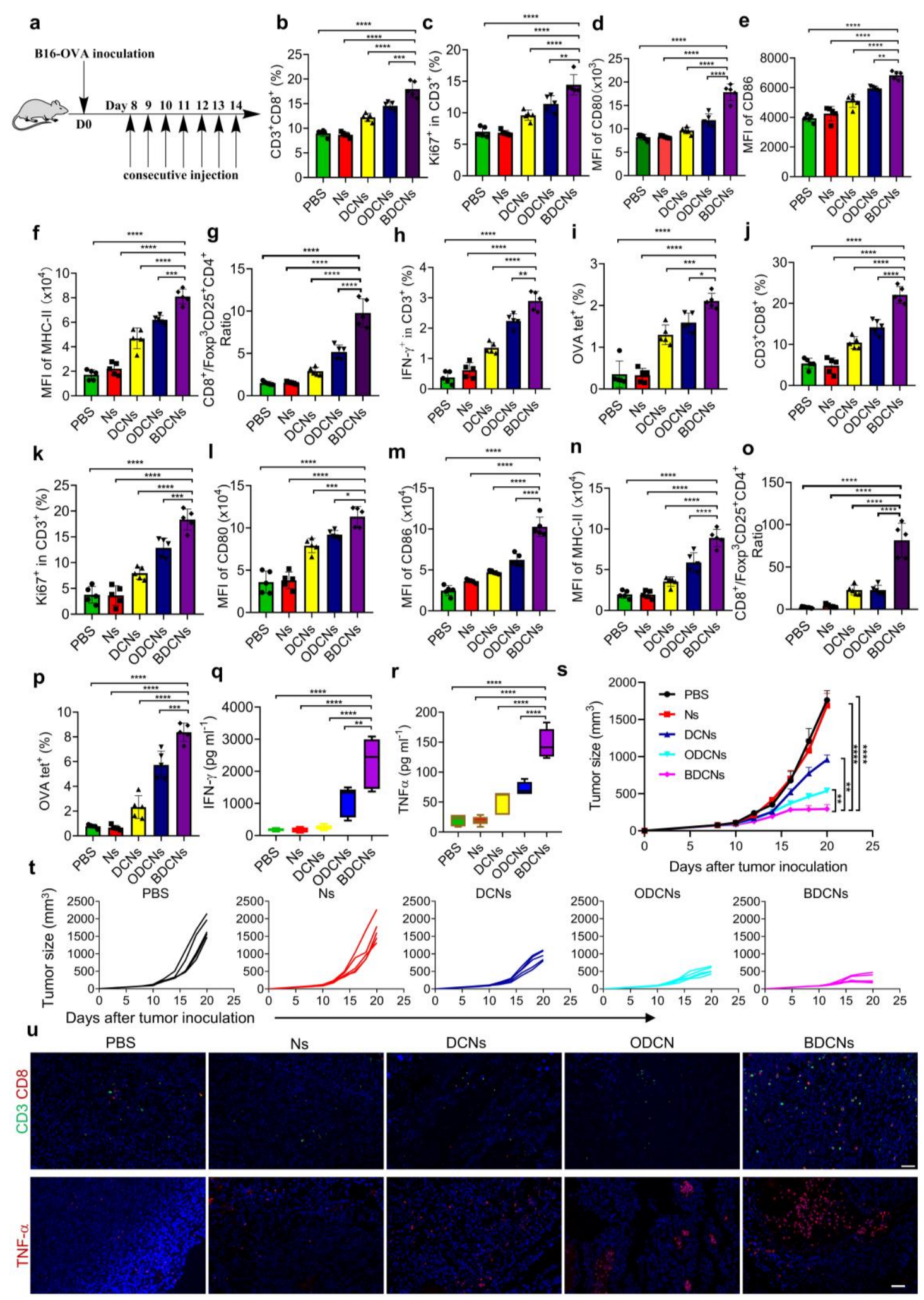

Figure 3. Antitumor immune responses induced by BNs in B16-OVA tumor-bearing mice. (a)

3 Experimental design for in vivo evaluation of antitumor immunity. B16-OVA tumor-bearing mice 4 were intravenously administrated with PBS, Ns, DCNs, ODCNs or BDCNs at predetermined time 5 points and sacrificed once tumor volume in control groups exceeded $2000 \mathrm{~mm}^{3}$. Percentages of (b) $6 \mathrm{CD}^{+} \mathrm{T}$ cells as effector $\mathrm{T}$ cells and (c) $\mathrm{Ki}^{+} 7^{+}$, mean fluorescence intensities of DC indicator (d) $7 \mathrm{CD} 80$, (e) $\mathrm{CD} 86$ and (f) MHC-II, (g) $\mathrm{CD} 8^{+} / \mathrm{Foxp} 3{ }^{+} \mathrm{CD} 25^{+} \mathrm{CD} 4^{+}$ratio, (h) IFN- $\gamma^{+}$and (i) OVA 
1 tetramer in the spleen. Percentages of (j) $\mathrm{CD}^{+} \mathrm{T}$ cells as effector $\mathrm{T}$ cells and (k) Ki67 ${ }^{+}$, mean 2 fluorescence intensities of DC indicator (l) CD80, (m) CD86 and (n) MHC-II, (o) $3 \mathrm{CD}^{+} / \mathrm{Foxp}^{+} \mathrm{CD}^{+} 5^{+} \mathrm{CD}^{+}$ratio, and (p) $\mathrm{OVA}^{+}$tet $^{+}$in the tumor. Levels of (q) IFN- $\gamma$ and (r) TNF- $\alpha$ 4 in the serum. (s) Average and (t) individual tumor growth curves. (u) Immunofluorescence staining 5 images of tumor tissue sectioned from the treated mice. $\mathrm{CD}^{+} \mathrm{T}$ cells (green channel), $\mathrm{CD} 8^{+} \mathrm{T}$ cells 6 (red channel), and TNF- $\alpha$ (red channel) were separately labeled. Scale bar: $50 \mu \mathrm{m}$. Error bars 7 represent the standard deviation $(n=5)$. Significance was assessed using One-way ANOVA with 8 Dunnett's post-hoc test. $* * * * p<0.0001, * * * p<0.001,{ }^{* *} p<0.01$, and $* p<0.05$. 

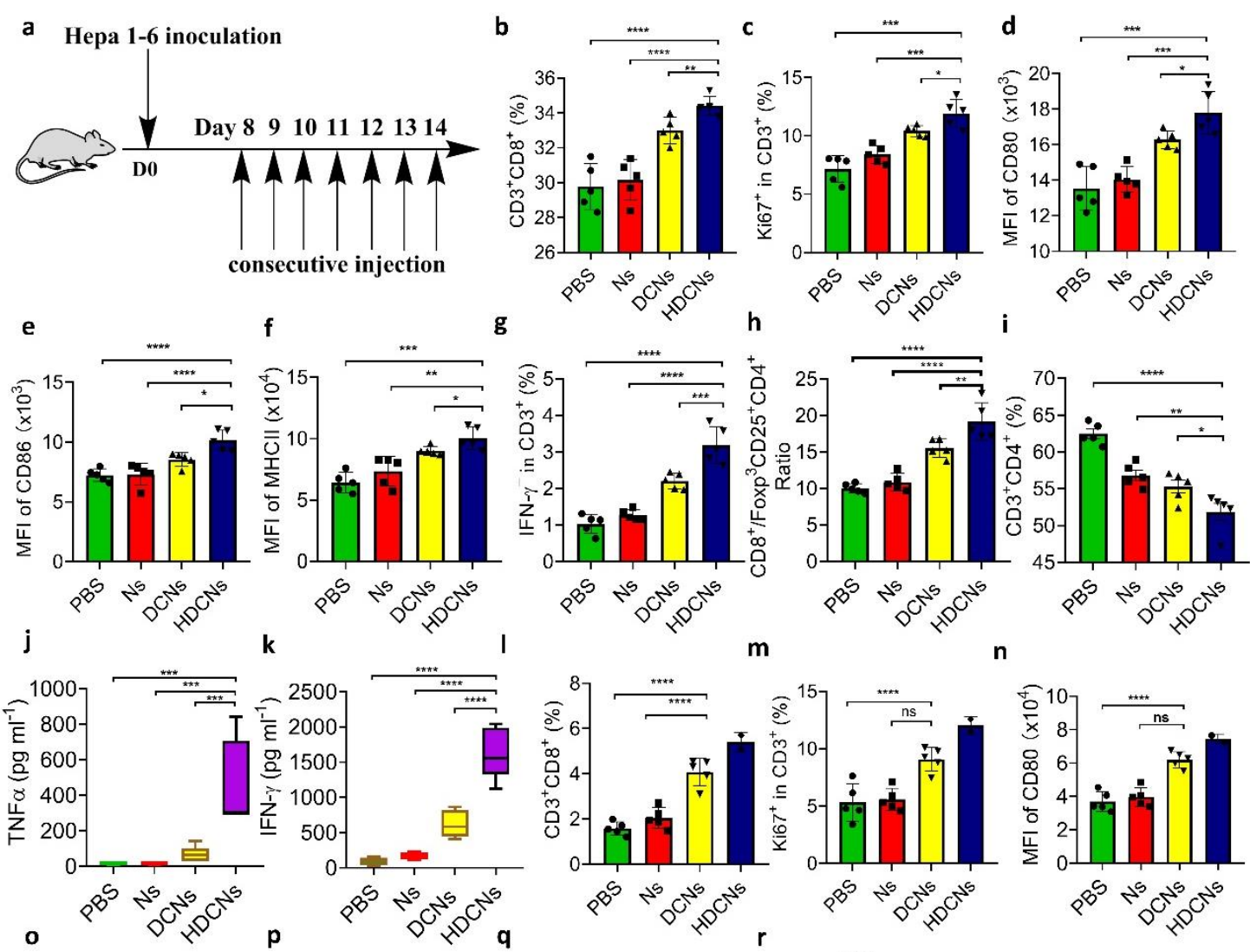

m
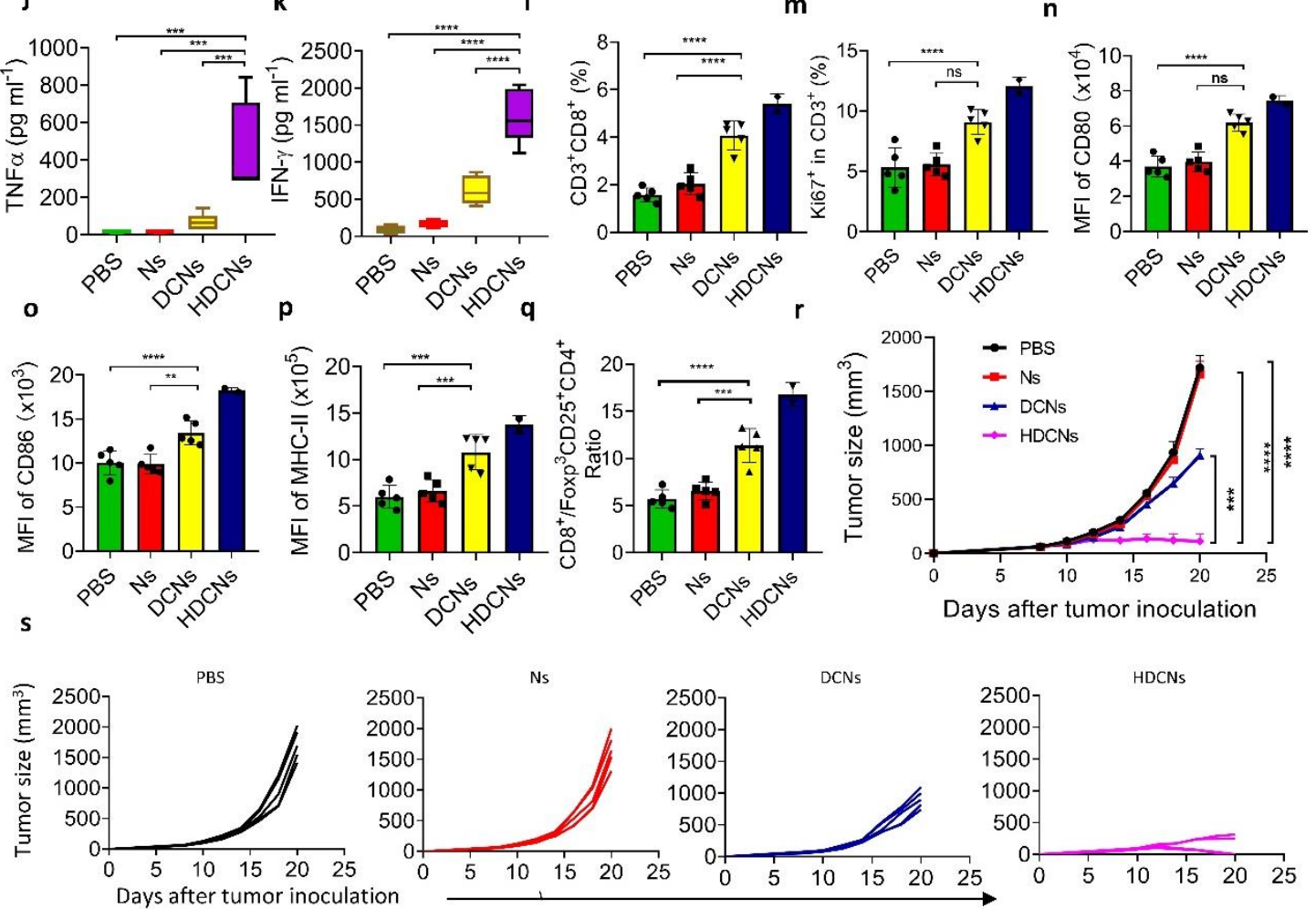

PBS

Ns

DCNs
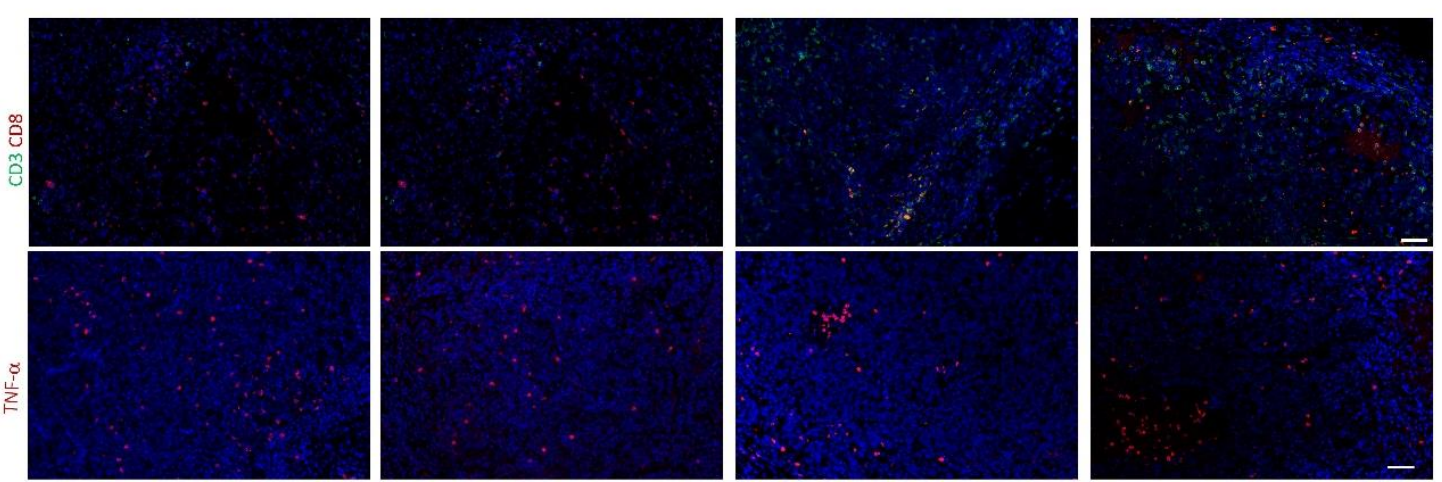
1 Figure 4. Antitumor immune responses elicited by BNs in Hepa 1-6 tumor-bearing mice. (a)

2 Experimental design for in vivo assessment of antitumor immune responses. Hepa 1-6 tumor3 bearing mice were intravenously injected with PBS, Ns, DCNs, or HDCNs at the indicated time 4 points and sacrificed once tumor volume in control groups exceeded $2000 \mathrm{~mm}^{3}$. Percentages of (b) $5 \mathrm{CD}^{+} \mathrm{CD}^{+}$and (c) $\mathrm{Ki}^{+} 7^{+} \mathrm{T}$ cells, mean fluorescence intensities of DC indicator (d) CD80, (e) CD86, 6 and (f) MHC-II, (g) IFN- $\gamma^{+}$, (h) $\mathrm{CD}^{+} / \mathrm{Foxp}^{+} \mathrm{CD} 25^{+} \mathrm{CD} 4^{+}$ratio and (i) $\mathrm{CD} 3^{+} \mathrm{CD} 4^{+}$in the spleen. 7 Expression of (j) TNF- $\alpha$ and (k) IFN- $\gamma$ in the serum. Percentages of (l) $\mathrm{CD} 3^{+} \mathrm{CD} 8^{+}$and (m) $\mathrm{Ki}^{+} 7^{+}$ 8 T cells, mean fluorescence intensities of DC indicator (n) CD80, (o) CD86, and (p) MHC-II, and (q) $9 \mathrm{CD}^{+} / \mathrm{Foxp}^{+} \mathrm{CD}^{+} 5^{+} \mathrm{CD} 4^{+}$ratio in the tumor tissue. (r) Average and (s) individual tumor growth 10 profiles. ( $\mathrm{t}$ ) Immunofluorescence staining images of tumor sampled from the treated mice. $\mathrm{CD}^{+} \mathrm{T}$ 11 cells (green channel), $\mathrm{CD}^{+} \mathrm{T}$ cells (red channel), and TNF- $\alpha$ (red channel) were labeled 12 individually. Scale bar: $50 \mu \mathrm{m}$. Error bars represent the standard deviation $(n=5)$. Significance was 13 assessed using One-way ANOVA with Dunnett's post-hoc test. $* * * * p<0.0001$, *** $p<0.001$, ** $p$ $14<0.01$, and $* p<0.05$. 


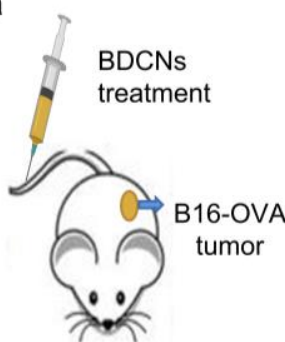

b

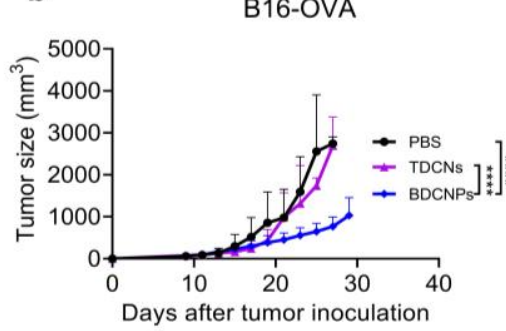

d

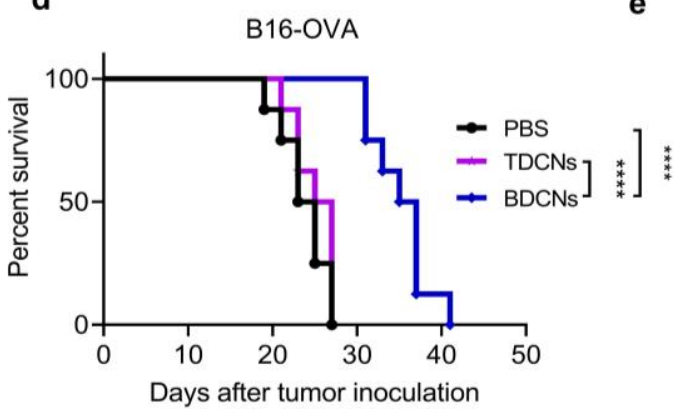

TC-1

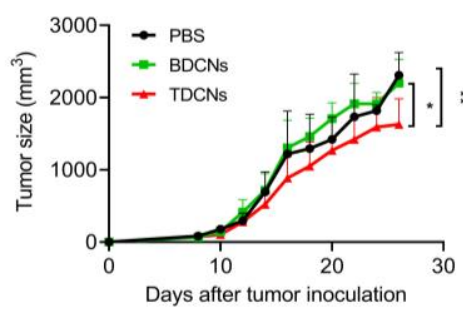

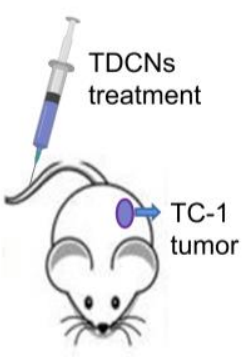

BDCNs

treatment

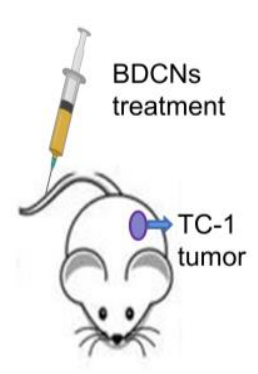

c

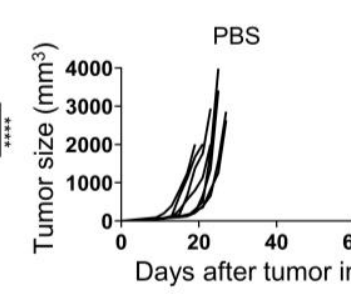

e

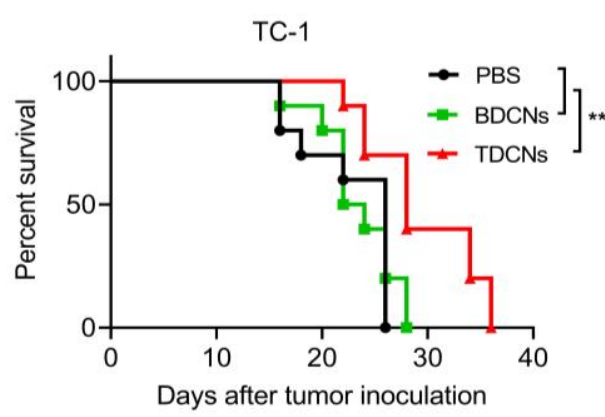

Figure 5. Cross-validation of BNs-leveraged antitumor immunity. (a) Experimental design for in vivo validation of antigen-specific antitumor immunity. After inoculation with $3 \times 10^{6}$ cells of B16-OVA or TC-1 on day 0 , mice were daily treated with PBS, TDCNs or BDCNs for 5 days after the tumor size reached 50 100 $\mathrm{mm}^{3}$. (b) Average and (c) individual tumor growth profiles of B16OVA tumor-bearing mice. Survivals of (d) B16-OVA and (e) TC-1 tumor-bearing mice after treatment. (f) Average and (g) individual tumor growth curves of TC-1 tumor-bearing mice. Error

8 bars represent the standard deviation $(n=8-10)$. Significance was assessed using One-way ANOVA

9 with Dunnett's post-hoc test. Survival rates were analyzed using the log-rank test. $* * * * p<0.0001$,

$10 \quad * * * p<0.001, * * p<0.01$, and $* p<0.05$. 

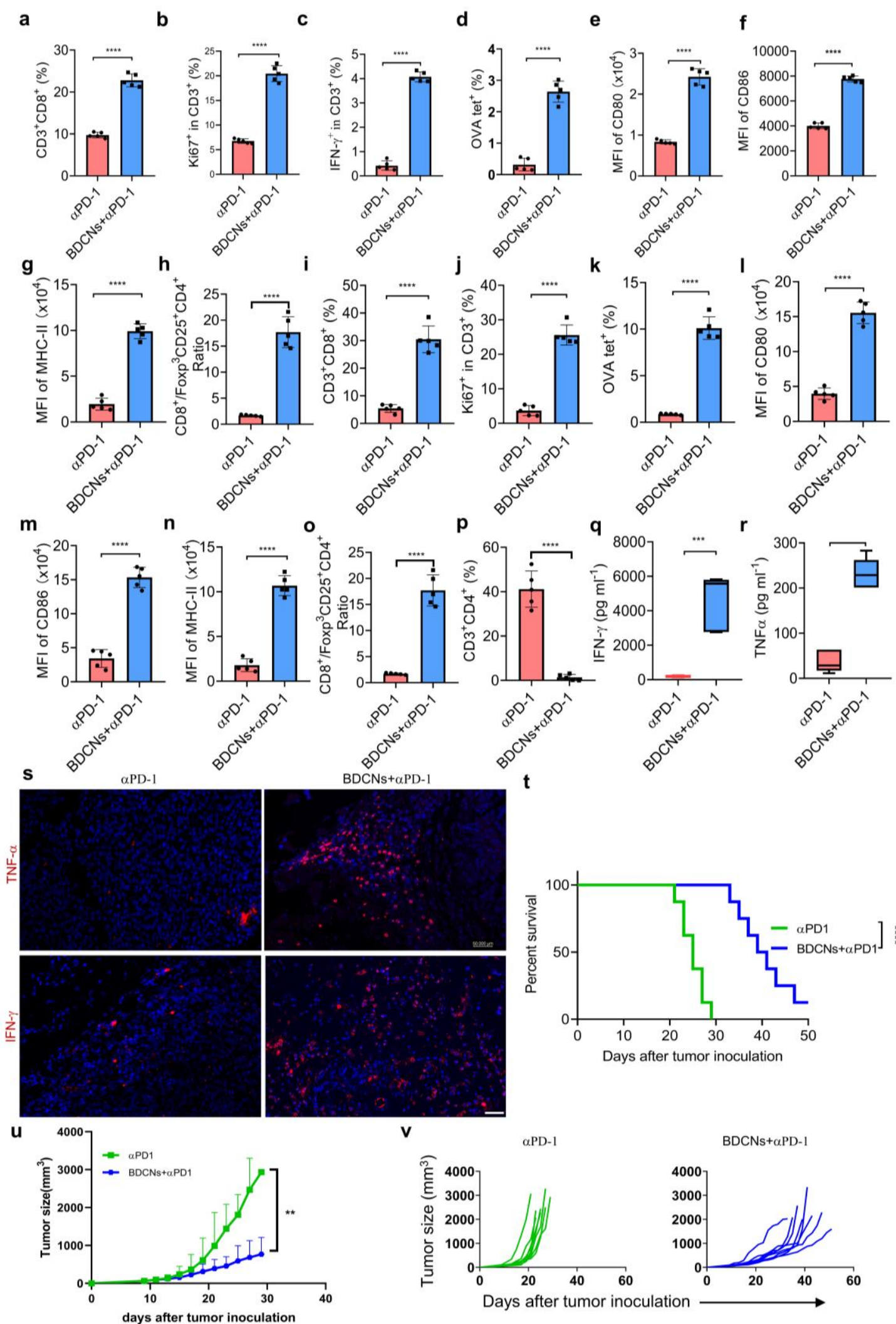

2 Figure 6. Combination of BDCNs with $\alpha$ PD-1 in B16-OVA tumor-bearing mice. After 3 inoculation with B16-OVA on day 0 , the mice were treated with intraperitoneal administration of $4100 \mu \mathrm{g} \alpha \mathrm{PD}-1$ on day $8,9,11,13$ and 15 along with daily tail vein injection of BDCNs through day 
18 to 14 . Equivalent free $\alpha$ PD-1 was used as a control. Mice were sacrificed for sampling once tumor 2 volume in the control group exceeded $2000 \mathrm{~mm}^{3}(n=5)$. Percentages of (a) $\mathrm{CD}^{+} \mathrm{CD}^{+}$and (b) $3 \mathrm{Ki}^{+} 7^{+} \mathrm{T}$ cells, (c) IFN- $\gamma^{+}$, (d) OVA tetramer, mean fluorescence intensities of DC indicator (e) CD80, 4 (f) $\mathrm{CD} 86$ and (g) $\mathrm{MHC}$-II, and (h) $\mathrm{CD}^{+} / \mathrm{Foxp}^{+} \mathrm{CD} 25^{+} \mathrm{CD} 4^{+}$ratio in the spleen tissue. Percentages 5 of (i) $\mathrm{CD}^{+} \mathrm{CD}^{+}$and (j) $\mathrm{Ki}^{+} 7^{+} \mathrm{T}$ cells, (k) OVA tetramer, mean fluorescence intensities of DC 6 indicator (l) $\mathrm{CD} 80$, (m) $\mathrm{CD} 86$ and (n) MHC-II, (o) $\mathrm{CD}^{+} / \mathrm{Foxp} 3{ }^{+} \mathrm{CD} 25^{+} \mathrm{CD} 4^{+}$ratio and (p) $7 \mathrm{CD}^{+} \mathrm{CD}^{+}$in tumor site. Concentrations of (q) IFN- $\gamma$ and (r) TNF- $\alpha$ in the serum. (s) 8 Immunofluorescence staining images of tumor tissue sampled from the treated mice. Both TNF- $\alpha$ 9 and IFN- $\gamma$ were labeled as red. Scale bar: $50 \mu \mathrm{m}$. (t) Survival as well as (u) average and (v) individual 10 tumor growth curves of mice treated with the combination of BDCNs with $\alpha$ PD-1 ( $n=8-10)$. 11 Significance was assessed using One-way ANOVA with Dunnett's post-hoc test. Survival rates 12 were analyzed using the log-rank test. $* * * * p<0.0001$ and $* * * p<0.001$ 

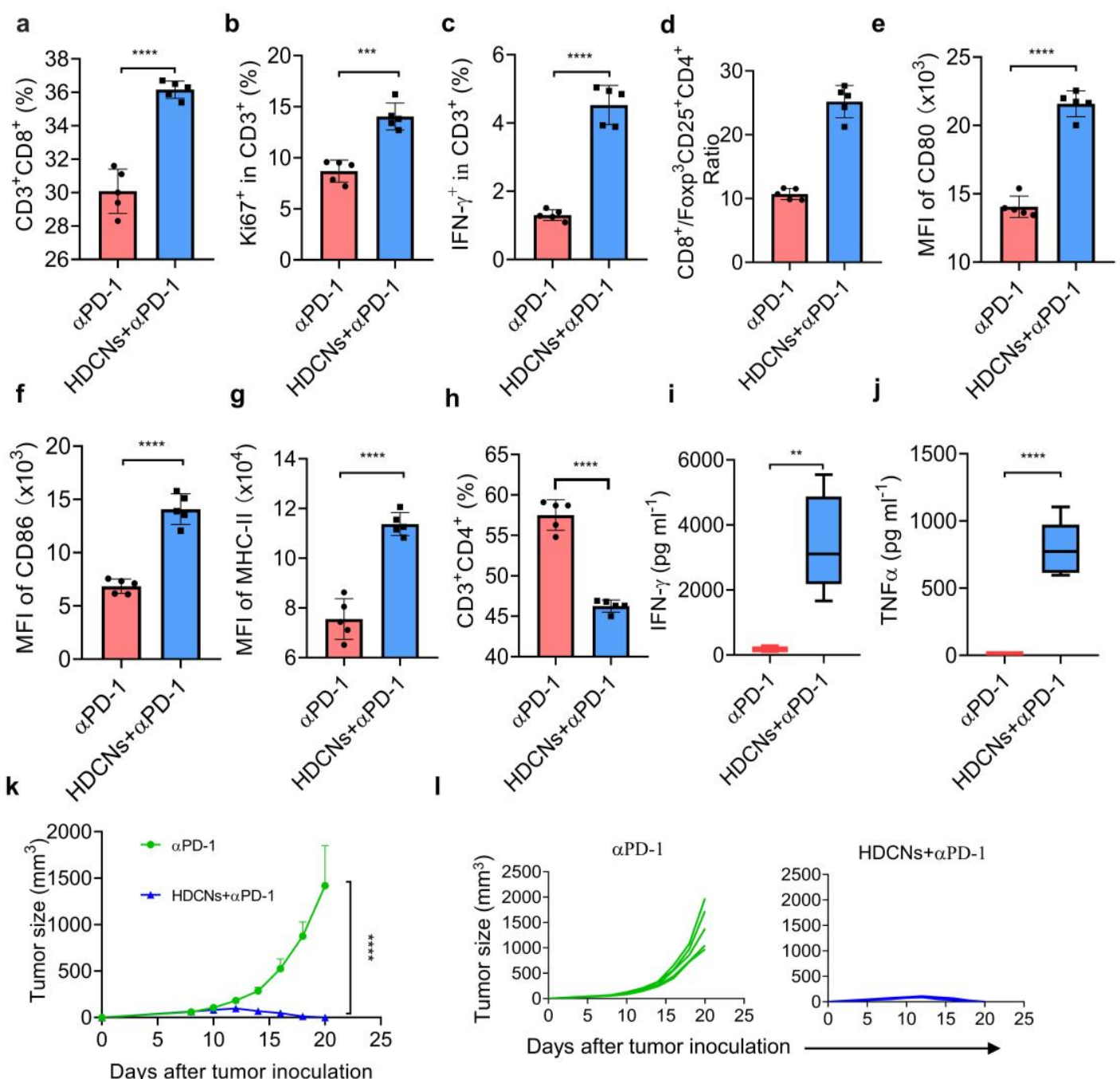

Figure 7. Combination of HDCNs with $\alpha$ PD-1 in Hepa 1-6 tumor-bearing mice. After inoculation with Hepa 1-6 on day 0, the mice were treated with intraperitoneal administration of

$4100 \mu \mathrm{g} \alpha \mathrm{PD}-1$ on day $8,9,11,13$ and 15 along with daily tail vein injection of HDCNs through day

58 to 14 . Equivalent free $\alpha \mathrm{PD}-1$ was used as a control. Mice were sacrificed for sampling once tumor 6 volume in the control group exceeded $2000 \mathrm{~mm}^{3}$. Percentages of (a) $\mathrm{CD}^{+} \mathrm{T}$ cells as effector T cells, (b) $\mathrm{Ki} 67^{+}$, (c) IFN- $\gamma^{+}$, mean fluorescence intensities of DC indicator (d) $\mathrm{CD} 8^{+} / \mathrm{Foxp} 3^{+} \mathrm{CD} 25^{+} \mathrm{CD} 4^{+}$ 8 ratio, (e) $\mathrm{CD} 80$ and (f) CD86, and (g) MHC-II in the spleen. Levels of (h) $\mathrm{CD}^{+} \mathrm{CD}^{+}$, (i) IFN- $\gamma$ and 9 (j) TNF- $\alpha$ in the serum. (k) Average and (l) individual tumor growth curves of the treated mice. 10 Error bars represent the standard deviation $(n=5)$. Significance was assessed using One-way 11 ANOVA with Dunnett's post-hoc test. $* * * * p<0.0001$, ***p $p 0.001$ and $* * p<0.01$. 


\section{Supplementary Files}

This is a list of supplementary files associated with this preprint. Click to download.

- SupplementarylnformationRevised.pdf 